\title{
Transient Analysis for Wireless Power Control
}

\author{
Maryam Fazel $^{1}$ Dennice Maynard Gayme ${ }^{1}$ Mung Chiang ${ }^{2}$ \\ ${ }^{1}$ Control and Dynamical Systems, Caltech. $\quad{ }^{2}$ Electrical Engineering, Princeton University.
}

\begin{abstract}
Power control mitigates interference and maintains required QoS levels in cellular wireless networks. An important class of distributed power control (DPC) was proposed by Foschini and Miljanic in 1993, with many variants developed since. Almost all related work focuses on the equilibrium and asymptotic convergence properties. However, for many applications transient behavior is more important. If a link's SIR drops below a critical threshold for too long, the connections over this link will be dropped, rendering the entire concept of equilibrium resource allocation meaningless. This paper proposes a systematic approach to the analysis of transient properties of DPC algorithms, in particular Foschini-Miljanic, based on tools from control theory. Analytically, we present a sufficient condition to ensure that after links reach their minimum SIR levels, their SIR requirements can be guaranteed for future time steps. Computationally, we pose this problem as verifying the invariance of certain regions in the SIR space, which for the basic DPC algorithm can be cast as a Linear Program (LP). Furthermore, using insights gained from the analysis, we propose a preliminary design framework for new iterative power control schemes.
\end{abstract}

Keywords: Invariant sets, Lyapunov functions, Power control, Wireless network.

\section{INTRODUCTION}

Power control in both voice and data cellular wireless networks is an important interference mitigation mechanism that has been extensively studied since the early 1990s. Many iterative [5], [3] and distributed [5], [6], [9] algorithms have been proposed; some of these converge to a globally optimal power allocation, while others converge to a Nash equilibrium. In all cases, efficiency and fairness of power allocation and Signal-Interference-Ratio (SIR) configuration at equilibrium have been the focus.

In this paper, we shift attention to the transients of power control. Indeed, while equilibrium properties are often more analytically tractable, they do not capture some of the more important issues in practical network operations. At the application connection level, dipping below a minimum SIR threshold during the transient phase of an iterative algorithm could cause the connection to disappear, thus rendering the whole concept of equilibrium SIR meaningless. Furthermore, the transient phase dominates the entire operating range when network dynamics vary at a similar time scale as the algorithm convergence speed.

As a starting point for a systematic study of transient behaviors in wireless power control, we consider the celebrated iterative algorithm of Foschini and Miljanic [5]. There are many transient properties worth examining, this paper mainly focuses on providing a guarantee that once a link is active, it retains the SIR level required to keep it active. Active link protection (and providing SIR guarantees in general) can be thought of in terms of invariance of regions in the SIR space. We use tools from control theory to obtain an analytic condition for providing SIR guarantees across all users. We also present a computational approach to verifying the invariance of the same SIR region via Linear Programming (LP). Then, turning from analysis to design, we present preliminary results on how to modify the basic algorithm to improve its transient behavior.

In general, we believe that a similar shift of research focus from asymptotic convergence to transient properties, including invariance, is desirable in many other research areas involving iterative resource allocation algorithms, such as congestion control, medium access, and buffer allocation. This paper is an initial step along this research direction. Often in current practice unpredictable and undesirable transient behaviors are treated through ad hoc means, and one of our goals is to illustrate how tools from control theory can help provide a systematic approach.

The paper is organized as follows; the following section introduces the problem setting, and provides a review of the Foschini-Miljanic algorithm. In section III the analytical results are presented along with a discussion of what they imply for link SIR guarantees. In section IV, we express the problem of checking the invariance of certain SIR regions as an LP, which can then be readily solved. The analytic results are verified by simulation in section $V$. New design of power control algorithm based on these insights is outlined in section VI, before we discuss some directions for future research in section VII.

Notation. Vectors are represented by letters in boldface, matrices are represented by capital letters and the $i^{t h}$ entry of $\mathbf{x}$ is denoted $x_{i}$.

\section{BACKGROUND AND RELATED WORK}

We consider a network of $n$ interfering links, each link consisting of a logical transmitter-receiver pair. This could, for example, model $n$ uplinks in a cellular network. Signalto-Interference-Ratio (SIR) of the $i^{\text {th }}$ link is denoted by

$$
r_{i}=\frac{G_{i i} p_{i}}{\sum_{j \neq i} G_{i j} p_{j}+\eta_{i}}, \quad i=1, \ldots, n
$$

where $G_{i j}>0$ is the power gain (path loss) from the transmitter of the $j^{\text {th }}$ link to the receiver of the $i^{\text {th }}$ one, $p_{i}$ is the power of the transmitter of the $i^{t h}$ link, $\eta_{i}$ is the thermal noise power at its receiver. Each link has a threshold SIR requirement $\alpha_{i}$ to maintain its connection through a minimum level of proper decoding, i.e., the link 
disappears if $r_{i}<\alpha_{i}$. A target SIR level of $\gamma_{i} \geq \alpha_{i}$ can be assigned through QoS provisioning to provide a 'safety margin'. Using (1) the inequalities $r_{i} \geq \gamma_{i}, \forall i$ (i.e., all links meet or exceed their target SIR requirements) can be written in matrix form as

$$
\left(I-D_{\gamma} F\right) \mathbf{p} \geq D_{\gamma} \mathbf{u}, \quad \mathbf{p}>0,
$$

where $\mathbf{p} \in \mathbb{R}^{n}$ is the power vector, $\mathbf{u} \in \mathbb{R}^{n}$ is the (normalized) noise vector, i.e., $u_{i}=\frac{\eta_{i}}{G_{i i}}, D_{\gamma}$ is a diagonal matrix with $\gamma_{i}$ s on its diagonal, and $F \in \mathbb{R}^{n \times n}$ is the matrix of cross-link power gains,

$$
F_{i j}= \begin{cases}0, & i=j, \\ \frac{G_{i j}}{G_{i i}}, & i \neq j,\end{cases}
$$

where $i, j=1, \ldots, n$. The following is a standard result on whether a given set of target SIRs are feasible.

Fact 1: Existence of a feasible power vector [3], [5]. Let $\mathcal{P}=\left\{\mathbf{p} \mid \mathbf{p}>0,\left(I-D_{\gamma} F\right) \mathbf{p} \geq D_{\gamma} \mathbf{u}\right\}$. The following statements are equivalent:

(i) There exists $\mathbf{p} \in \mathcal{P}$,

(ii) $\rho\left(D_{\gamma} F\right)<1$, where $\rho$ denotes the maximum modulus eigenvalue,

(iii) $\left(I-D_{\gamma} F\right)^{-1}$ exists and is positive componentwise. Based on the above, the iteration

$$
\mathbf{p}(k+1)=D_{\gamma} F \mathbf{p}(k)+D_{\gamma} \mathbf{u},
$$

introduced in [5] by Foschini and Miljanic, converges to $\mathbf{p}^{*}=\left(I-D_{\gamma} F\right)^{-1} D_{\gamma} \mathbf{u}$ when $\mathbf{p}^{*}$ exists. This iteration can alternatively be expressed as

$$
p_{i}(k+1)=\frac{\gamma_{i}}{r_{i}(k)} p_{i}(k), \quad i=1, \ldots, n,
$$

where only local SIR measurements $r_{i}(k)$ are needed for the update. Since the update decision at each link is performed independently, based on information collected on it exclusively, the algorithm is referred to as a Distributed Power Control algorithm (DPC). Many other variants of this DPC have been proposed over the years. In particular, this algorithm has been extended for other network models, e.g., [7], [8], [9] examined asynchronous implementation, bursty transmissions, and multiclass traffic; [13], [14] considered joint power and base station assignment, and [1], [2] studied admission control with power control. As a key paper that started to examine non-equilibrium properties of DPC algorithms, [3] tackled the issue of protecting active links while new links are introduced, by modifying the FoschiniMiljanic algorithm to include two different update rules for active and inactive links.

The present work further shifts the attention to what happens to DPC before reaching the equilibrium. We use tools from control theory to study aspects of the dynamics and the evolution of the SIRs in the Foschini-Miljanic algorithm. Using these tools we not only provide analytical results on some SIR invariant regions for the FoschiniMiljanic algorithm but also provide computational methods to evaluate invariance for a particular problem setup. Such analysis not only provides a deeper understanding of DPCs, but also helps with designing new power control algorithms with better SIR guarantees and transient properties. This type of design approach is more systematic than the current practice of treating undesirable transient behaviors through ad hoc means (e.g., raising target SIR levels and hoping for fast convergence or averaging effects of network dynamics). We set up and discuss a general framework for such designs in section VI.

\section{INVARIANT REGIONS IN SIR SPACE}

Consider an autonomous discrete-time dynamical system $\mathbf{x}(k+1)=f(\mathbf{x}(k)), \mathbf{y}(k)=g(\mathbf{x}(k))$, with initial state $\mathbf{x}(0)$. The state of the system and the output at time $k$ are $\mathbf{x}(k) \in \mathbb{R}^{n}$ and $\mathbf{y}(k) \in \mathbb{R}^{m}$ respectively. A subset of the output space is called invariant if once the output enters this set, it remains there for all future time steps [12], i.e.

$$
\mathbf{y}(k) \in \mathcal{S} \Rightarrow \mathbf{y}(k+1) \in \mathcal{S}, \quad \forall k .
$$

For the Foschini-Miljanic algorithm we call the power $p_{i}(k)$ the state of each link $i$ at time $k$ and refer to the corresponding SIR $r_{i}(k)$ as the output, so the system is described by equations (3) and (1). We are interested in the transient behavior of the output (the SIRs) of this system. To study this problem we begin by addressing questions such as: if all links achieve their minimum SIR, will they continually stay above this level for all future time? Also, if an individual link achieves its minimum SIR, under what conditions will it remain above this level? Both of these questions can be addressed though determining if there are corresponding invariant regions in the SIR space. Throughout this section we assume that $\rho\left(D_{\gamma} F\right)<1$, so the $\gamma_{i}$ s are always feasible SIR targets.

\section{A. SIR guarantees}

We begin with the first question posed in the previous section: Assuming all links have already achieved their minimum SIR level, under what conditions can we guarantee that they retain this SIR level for all subsequent time steps? This type of guarantee could be sought for example when all the links are powering up at the same time.

Proposition 1: Common ratio condition. A sufficient condition for $r_{i}(k) \geq \alpha_{i}, \forall i \Longrightarrow r_{i}(k+1) \geq \alpha_{i}, \forall i$ is that there is a constant $\delta>0$ such that

$$
\frac{\gamma_{i}}{\alpha_{i}}=\delta, \forall i
$$

Proof: The invariance relation can be written using (2),

$$
\begin{aligned}
& \left(I-D_{\alpha} F\right) \mathbf{p}(k)-D_{\alpha} \mathbf{u} \geq 0 \Longrightarrow \\
& \left(I-D_{\alpha} F\right) \mathbf{p}(k+1)-D_{\alpha} \mathbf{u} \geq 0 .
\end{aligned}
$$

Substituting for $\mathbf{p}(k+1)$ in (7) yields

$$
\left(I-D_{\alpha} F\right) D_{\gamma} F \mathbf{p}(k)+\left[\left(I-D_{\alpha} F\right) D_{\gamma}-D_{\alpha}\right] \mathbf{u} \geq 0 .
$$

Noting $D_{\gamma}=\delta D_{\alpha} \Rightarrow D_{\alpha} F D_{\gamma}=D_{\gamma} F D_{\alpha}$ and rearranging (8)

$$
\delta D_{\alpha} F\left[\left(I-D_{\alpha} F\right) \mathbf{p}(k)-D_{\alpha} \mathbf{u}\right]+(\delta-1) D_{\alpha} \mathbf{u} \geq 0 .
$$


The condition in (6), along with the fact that the matrix $D_{\alpha} F$ is componentwise nonnegative and the assumption that $\delta \geq 1$, implies that the above inequality holds for any channel gains $G_{i j}$ and noise powers $\eta_{i}$. Thus, the shaded region in figure 1 is invariant.

We can think of $\frac{\gamma_{i}}{\alpha_{i}}=\delta$ as the 'safety margin' because it allows for each link to be a factor $\delta$ above its minimum required SIR. So, the common ratio condition simply means that all links have the same safety margin.

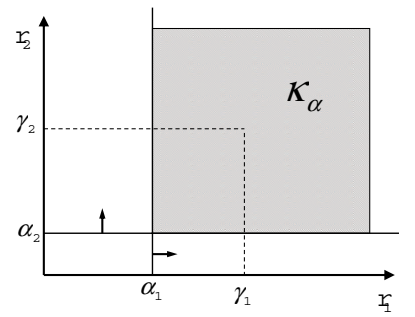

Fig. 1. Regions in SIR space whose invariance is discussed

Remark 1: Invariant cones. Proposition 1 states that, for any $\gamma, F$, and $\mathbf{u}$ that satisfy $\rho\left(D_{\gamma} F\right)<1$, particular cones in the SIR space are invariant. These cones are copies of the nonnegative orthant $\mathbb{R}_{+}^{n}$ shifted by a constant $\frac{1}{\delta}$ times the vector $\gamma$, which can be parameterized by $\delta$ as $\mathcal{K}_{\delta}=\mathbb{R}_{+}^{n}+\frac{1}{\delta} \gamma$, for all $\delta \geq 1$. The cones $\mathcal{K}_{\delta}$ can be extended to more general rectangular regions, as discussed in section III-B.

The common ratio condition is only a sufficient condition, but it has useful implications even for cases that do not satisfy it, as shown in section III-C. ${ }^{1}$

A natural question is whether there are similar conditions under which any link $i$ that reaches its minimum SIR of $\alpha_{i}$ stays above this level, regardless of the SIRs of the other links. This corresponds to the invariance of the half-space regions indicated by the arrows in figure 1 , and would require that

$$
\begin{aligned}
& \left(\mathbf{e}_{i}-\alpha_{i} \mathbf{f}_{i}\right)^{T} \mathbf{p}(k)-\alpha_{i} u_{i} \geq 0 \Rightarrow \\
& \quad\left(\mathbf{e}_{i}-\alpha_{i} \mathbf{f}_{i}\right)^{T} D_{\gamma} F \mathbf{p}(k)+\left[\left(\mathbf{e}_{i}-\alpha_{i} \mathbf{f}_{i}\right)^{T} D_{\gamma}-\alpha_{i} e_{i}\right] \mathbf{u} \geq 0,
\end{aligned}
$$

where $\mathbf{e}_{i}$ is a vector with 1 in its $i^{\text {th }}$ entry and zero elsewhere, and $\mathbf{f}_{i}$ denotes the $i^{\text {th }}$ row of the matrix $F$.

The answer is negative even for the case where $\frac{\gamma_{i}}{\alpha_{i}}=\delta, \forall i$. In this case the righthand side expression can be simplified to

$$
\delta \mathbf{f}_{i}^{T}\left[\left(I-D_{\alpha} F\right) \mathbf{p}(k)-D_{\alpha} \mathbf{u}\right]+(\delta-1) u_{i} \geq 0 .
$$

All we know from the lefthand side of (9) is the nonnegativity of the $i^{t h}$ entry in the vector $\left(I-D_{\alpha} F\right) \mathbf{p}(k)-D_{\alpha} \mathbf{u}$. The term in square brackets involves other entries in this vector, which are negative for links that have not reached their minimum SIR. Therefore, in general, nothing can be concluded about

\footnotetext{
${ }^{1}$ Note that the case considered in section 2 of [3] $\left(\alpha_{i}=\gamma_{i}\right)$ is a special case of the Proposition 1. Furthermore, the active link update, which is part of their ALP/DPC algorithm, also assumes a fixed ratio between the target SIR and the minimum SIR.
}

the sign of the overall expression, and invariance of the halfspaces cannot be guaranteed for a general $F$ and $\mathbf{u}$ without imposing conditions on the powers of other links. To have such system-independent guarantee, some modifications to the basic algorithm are needed, as will be discussed towards the end of this paper.

\section{B. Lyapunov function interpretation}

The invariant cones discussed above are reminiscent of the level sets of a Lyapunov function. Indeed, the cones $\mathcal{K}_{\delta}$ can be extended to invariant rectangles, which form the level sets of a Lyapunov function. The following lemma describes the associated rectangles.

Lemma 1: For any $\delta>1$,

$$
\begin{aligned}
\left|r_{i}(k)-\gamma_{i}\right| \leq \gamma_{i}\left(1-\frac{1}{\delta}\right), \forall i \Rightarrow & \\
& \left|r_{i}(k+1)-\gamma_{i}\right|<\gamma_{i}\left(1-\frac{1}{\delta}\right), \forall i
\end{aligned}
$$

Proof: We first write the inequalities in terms of powers. From (2), $\left|r_{i}(k)-\gamma_{i}\right| \leq \gamma_{i}\left(1-\frac{1}{\delta}\right), \forall i$, is equivalent to

$$
\begin{aligned}
& {\left[I-\frac{1}{\delta} D_{\gamma} F\right] \mathbf{p}(k)-1 / \delta D_{\gamma} \mathbf{u} \geq 0 \text { and }} \\
& \quad\left[I-\left(2-\frac{1}{\delta}\right) D_{\gamma} F\right] \mathbf{p}(k)-\left(2-\frac{1}{\delta}\right) D_{\gamma} \mathbf{u} \leq 0,
\end{aligned}
$$

where the inequalities correspond to the lower and upper bounds on $r_{i}(k)-\gamma_{i}$, respectively. We want to show that these inequalities also hold at $k+1$. Consider the first inequality. Substituting for $\mathbf{p}(k+1)$ and rearranging the terms yields

$$
\begin{aligned}
& {\left[I-\frac{1}{\delta} D_{\gamma} F\right] \mathbf{p}(k+1)-\frac{1}{\delta} D_{\gamma} \mathbf{u}} \\
& =\left[I-\frac{1}{\delta} D_{\gamma} F\right]\left(D_{\gamma} F \mathbf{p}(k)+D_{\gamma} \mathbf{u}\right)-\frac{1}{\delta} D_{\gamma} \mathbf{u} \\
& =D_{\gamma} F\left(\left[I-\frac{1}{\delta} D_{\gamma} F\right] \mathbf{p}(k)-\frac{1}{\delta} D_{\gamma} \mathbf{u}\right)+\left(1-\frac{1}{\delta}\right) D_{\gamma} \mathbf{u} .
\end{aligned}
$$

The last expression is strictly positive for $\delta>1$ (if $\delta \geq 1$, this inequality is nonstrict), since the term in the parenthesis is nonnegative, and $D_{\gamma} F$ is nonnegative componentwise. We conclude that $r_{i}(k+1)-\gamma_{i}>\gamma_{i}\left(1-\frac{1}{\delta}\right)$. The upper bound can be proved similarly.

Thus, the rectangular regions shown in figure 2 are invariant, and form the level sets of the Lyapunov function that proves the convergence of $\mathbf{r}(k)$, (the SIRs), to $\gamma$.

Corollary 1: The invariant sets given in Lemma 1 are the level sets of the following Lyapunov function

$$
\begin{aligned}
\mathcal{V}(\mathbf{r}(k)) & =\max _{i} \frac{1}{\gamma_{i}}\left|r_{i}(k)-\gamma_{i}\right| \\
& =\left\|D_{\gamma}^{-1}(\mathbf{r}(k)-\gamma)\right\|_{\infty} .
\end{aligned}
$$

Remark 2: Bounds on SIR drops. The invariant rectangles shown in figure 2 indicate that at any time $k$, the possible SIR drops are always bounded by a known value. For example in figure $2, \mathbf{r}(k)$ lies on the boundary of one of the rectangles. 


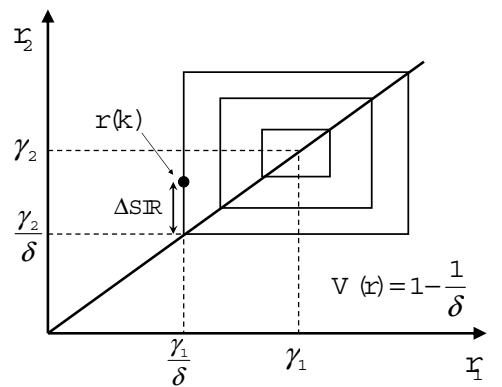

Fig. 2. The level sets of the Lyapunov function

Since this rectangle is invariant $\mathbf{r}(k+1)$ will also be inside this region. Therefore, drops in the SIR of each link, i.e., components of vector $\mathbf{r}(k)-\mathbf{r}(k+1)$, can by no larger than the componentwise distance of $\mathbf{r}(k)$ to the line $\frac{r_{i}}{\gamma_{i}}=\delta$; this distance is labeled $\triangle S I R$ in figure 2.

\section{$C$. The case where $\gamma \neq \delta \boldsymbol{\alpha}$ (links may have different 'safety margins')}

Even when the condition in Proposition 1 is not satisfied, that is the SIR 'safety margin' is not the same for all links, the result in Lemma 1 provides some conditions on the link SIR levels. Figure 3 illustrates a situation where the condition

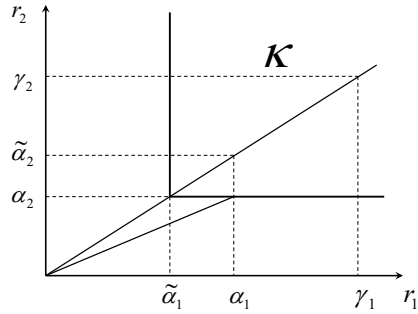

Fig. 3. The case where $\boldsymbol{\gamma} \neq \delta \boldsymbol{\alpha}$

from Proposition 1 is not satisfied (i.e. the vectors $\gamma$ and $\boldsymbol{\alpha}$ are not aligned). If we let $\tilde{\alpha}_{1}=\frac{\gamma_{1}}{\gamma_{2}} \alpha_{2}$, we know from Proposition 1 that the cone $\mathcal{K}$ with its vertex at $\left(\tilde{\alpha}_{1}, \alpha_{2}\right)$ is invariant. So once the SIR for link $2\left(r_{2}\right)$ enters this cone at time $K, r_{2}(k) \geq \alpha_{2}(k) \forall k>K$. Thus, to keep link 2 active, link 1 need only achieve an SIR of $\tilde{\alpha}_{1}$, which is lower than its SIR threshold $\left(\alpha_{1}\right)$. This property extends to $n$ links as follows.

Proposition 2: If $r_{i}(k) \geq \alpha_{i}$ and $r_{j}(k) \geq \frac{\gamma_{j}}{\gamma_{i}} \alpha_{i}$ for all $j \neq i$, then $r_{i}(k+1) \geq \alpha_{i}$ (i.e., link $i$ is protected).

Proof: The proof follows immediately from the fact that $\frac{\gamma_{j}}{\tilde{\alpha}_{j}}=\frac{\gamma_{i}}{\alpha_{i}}=\delta_{i}$, for all $j \neq i$, so $\gamma$ and $\tilde{\alpha}$ satisfy the common ratio condition.

Note that in figure 3 the cone that guarantees both SIRs are above their thresholds has its vertex at $\left(\alpha_{1}, \tilde{\alpha}_{2}\right)$, where $\tilde{\alpha}_{2} \geq$ $\alpha_{2}$. This means that, if we raise the SIR thresholds of other users, invariance is guaranteed (at the cost of consuming more power)

\section{InVARIANCE ANALYSis: LINEAR PROGRAMming} APPROACH

In this section we discuss how to computationally verify that all links remain active after they all achieve their threshold SIRs. Note that, here and in the sequel, $\mathbf{p}(k)$ is replaced by $\mathbf{p}$ for simplicity in notation.

Proposition 3: Given the system described by (3) and (1), the following conditions are equivalent

(i) $r_{i}(k) \geq \alpha_{i} \Rightarrow r_{i}(k+1) \geq \alpha_{i}, \quad \forall i$

(ii) The set

$$
\begin{aligned}
& \left\{\mathbf{p} \mid \mathbf{p} \geq 0,\left(I-D_{\alpha} F\right) \mathbf{p} \geq D_{\alpha} \mathbf{u},\right. \\
& \left.\left(I-D_{\alpha} F\right) D_{\gamma} F \mathbf{p}<\left[D_{\alpha}-\left(I-D_{\alpha} F\right) D_{\gamma}\right] \mathbf{u}\right\}
\end{aligned}
$$

is empty.

(iii) $\exists \boldsymbol{\lambda} \succeq 0$ such that $A^{T} \boldsymbol{\lambda}=\mathbf{0}, \boldsymbol{\lambda}^{T} \mathbf{c}<0$ where

$$
\begin{gathered}
A=-\left[\begin{array}{c}
I-D_{\alpha} F \\
{\left[D_{\alpha} F-I\right] D_{\gamma} F} \\
I
\end{array}\right], \text { and } \\
\mathbf{c}=-\left[\begin{array}{c}
D_{\alpha} \mathbf{u} \\
{\left[I-D_{\alpha} F\right] D_{\gamma} \mathbf{u}-D_{\alpha} \mathbf{u}+\varepsilon} \\
0
\end{array}\right] .
\end{gathered}
$$

and finding this $\boldsymbol{\lambda}$ is equivalent to solving the dual of Proof: the LP feasibility problem ${ }^{2}$ : find $\mathbf{p}$ such that $A \mathbf{p} \preceq \mathbf{c}$

(ii) $\Leftrightarrow$ (i): Emptiness of the set (11) implies $\forall \mathbf{p} \geq 0$ the region defined by $\left(I-D_{\alpha} F\right) \mathbf{p} \geq D_{\alpha} \mathbf{u}$ (at time $k$ ) and the complement of this region at time $(k+1)$ do not intersect; i.e., no vector exists that satisfies all conditions in (11) simultaneously. This means starting in the region guarantees remaining there for all future time.

(ii) $\Leftrightarrow$ (iii): Farkas' Lemma or LP duality [4] states that the LP: find $\mathbf{p}$ such that $A \mathbf{p} \preceq \mathbf{c}$ is infeasible by if and only if the dual problem: find $\boldsymbol{\lambda} \succeq 0$ such that $A^{T} \boldsymbol{\lambda}=$ $0, \boldsymbol{\lambda}^{T} \mathbf{c}<0$ is feasible. So, the solution to this problem provides a certificate of infeasibility for the primal.

\section{A. Numerical example}

We consider a case with four links, a power gain matrix

$$
G=\left[\begin{array}{cccc}
7.730 & 0.241 & 0.407 & 0.316 \\
0.263 & 7.883 & 0.165 & 0.247 \\
0.219 & 0.224 & 7.939 & 0.146 \\
0.184 & 0.0498 & 0.117 & 7.373
\end{array}\right],
$$

$\eta_{i}=10^{-2}$, and $\varepsilon=10^{-10}$. Selecting $\alpha=$ $[9.27,7.92,8.75,5.83]$ and $\gamma=[10,9,8.5,9.5]$ generates the vector of ratios $\frac{\gamma_{i}}{\alpha_{i}}=[1.187,1.2,1.2,1.2]$, (which does not satisfy the common ratio condition). To solve the LP we used, among the many existing solvers, the software package SeDuMi [11]. Solving the dual problem yields Lagrange multipliers $\boldsymbol{\lambda} \succeq 0$ that satisfy $\boldsymbol{\lambda}^{T}(\mathbf{c}-A \mathbf{p}) \approx-1$ and provide a certificate of infeasibility for the LP.

Recall that the feasibility problem is finding $\mathbf{p}$ such that $\mathbf{c}-A \mathbf{p} \succeq 0$. Multiplying this it by $\boldsymbol{\lambda} \succeq 0$ would yield a positive number. So the equation $\boldsymbol{\lambda}^{T}(\mathbf{c}-\bar{A} \mathbf{p}) \approx-1$ provides

\footnotetext{
${ }^{2}$ The $\varepsilon$ in the $\mathbf{c}$ vector is to deal with the fact that in (11) there is a strict inequality whereas the standard form of an LP has a nonstrict inequality.
} 

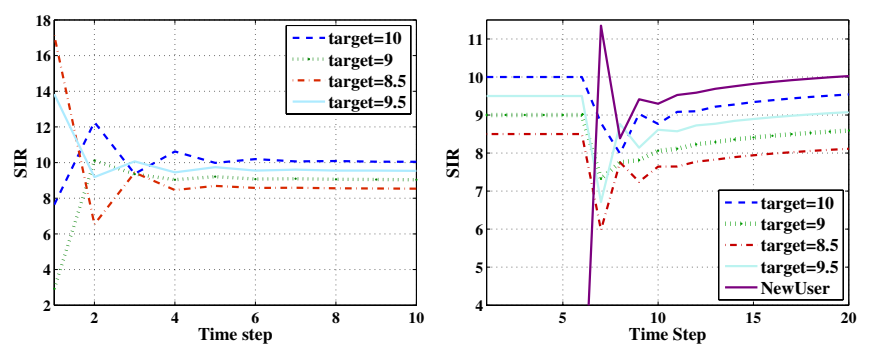

Fig. 4. Power Up Phase for 4 links Fig. 5. Adding a new user (link)

a contradiction that proves the LP is infeasible and thus the region $\{\mathbf{r}(k) \mid \mathbf{r}(k) \geq \boldsymbol{\alpha}\}$ is invariant. This proof method is a special case of a more general proof method in [10].

This example shows that the common ratio condition from Proposition 1 is not necessary in general; that is, invariance can hold without it.

\section{NUMERiCAL RESUlTS}

In this section we give a numerical example that demonstrates that Proposition 1 is valid both during startup and when a new user is added. These are the conditions that induce a disturbance on the system and thus the most likely to cause a link's SIR to drop. For the startup case the $G$ matrix and $\eta_{i}$ 's are the same as in the example IVA. The target SIR's for each link are given in the vector $\gamma=[10,9,8.5,9.5]$ as shown in figure 4, the minimum SIR for each link is set to $\boldsymbol{\alpha}=[8.43,7.5,7.08,7.92]$ which means that $\delta=1.2$ for this example. The initial power was set to $\mathbf{p}(0)=10^{-2}[6.83,2.13,8.39,6.29]$.

After the four links had reached and settled at their target SIR's we introduced a fifth link with an SIR target of 10.5 and corresponding $G_{i j}$ 's that keep the system feasible (i.e $\left.\rho\left(D_{\gamma} F\right)<1\right)$. The new link has a higher target SIR than all of the old links because this was thought to lead to a larger disturbance for the other links. The new power gain matrix is

$$
G=\left[\begin{array}{ccccc}
7.730 & 0.241 & 0.407 & 0.316 & 0.106 \\
0.263 & 7.883 & 0.165 & 0.247 & 0.151 \\
0.219 & 0.224 & 7.939 & 0.146 & 0.259 \\
0.184 & 0.0498 & 0.117 & 7.373 & 0.184 \\
0.155 & 0.0968 & 0.299 & 0.237 & 7.982
\end{array}\right]
$$

Figure 5 shows the effect of adding a new link. The new five link system has the minimum SIR vector $\boldsymbol{\alpha}=$ $[8.43,7.5,7.08,7.92,8.75]$. It is clear from the figure that once all of the links surpass their minimum values they remain above them for all time. In fact all of the links eventually converge to their target values. Note that in all figures in this and the following section SIRs are plotted on a linear scale.

\section{DESIGN FOR TRANSIENTS}

Given that we can compute conditions to quantify the transient behavior, an interesting question now is how we can use that knowledge to design a power control algorithm that limits undesirable behavior. This includes slow convergence during the startup phase and links dropping below their target SIR's when the network changes due to an external disturbance (e.g., the addition of a new link, or a change in the target SIR level of an active link). In general, control design (synthesis) is a difficult problem. However, one can reformulate the Foschini-Miljanic DPC into a more general framework (13) (also see (16)), which allows one to adjust the update rule based on the current state of the system. Here we present a preliminary study and an example of how this framework can be used. Systematic control design for this problem is a topic for future work.

Consider a generalization Foschini-Miljanic update (4) where the target SIR is time varying,

$$
p_{i}(k+1)=\frac{\gamma_{i}(k)}{r_{i}(k)} p_{i}(k) .
$$

This provides the following framework for general $\gamma$ updates

$$
\gamma_{i}(k+1)=f\left(r_{i}(k), p_{i}(k),\left\{\gamma_{i}(1), \ldots, \gamma_{i}(k)\right\}\right),
$$

which allows many choices for $\gamma_{i}(k+1)$ with each choice leading to a different algorithm. For example, the DPC/ALP algorithm of Bambos et al [3], which involves two separate modes for active and inactive links, can be viewed as a case of (13), with $\gamma_{i}(k)$ given by the piecewise linear function

$$
\gamma_{i}(k)= \begin{cases}\delta r_{i}(k) & \text { for } r_{i}(k)<\gamma_{i}^{*}, \\ \delta \gamma_{i}, & \text { for } r_{i}(k) \geq \gamma_{i}^{*},\end{cases}
$$

where $\gamma^{*}$ is the target SIR. In this case there is feedback based on $r_{i}(k)$ that allows each link to determine which mode they are in and perform a mode switch when it is appropriate.

In many cases it may not be desirable for each link to have to do an additional calculation to determine which mode they are operating in or to keep track of more than one update rule. There are many reasons that a user may need to switch from one mode to the other such as variations in network conditions, changes in target SIR's or the addition of new users in a cooperative network. In (15) a simple alternative that does not require switching between two operation modes is proposed, where $\gamma_{i}$ is updated based on how far it is from its target value.

$$
\gamma_{i}(k+1)=\gamma_{i}^{*}-\kappa\left|\gamma_{i}^{*}-\gamma_{i}(k)\right|,
$$

where $\kappa$ is an arbitrary constant that affects the rate of convergence and the sensitivity of the system to disturbances. A low $\kappa$ results in a disturbance having a larger effect but a faster convergence rate whereas a larger $\kappa$ makes the SIR levels less likely to drop when a new user is added. This tradeoff between sensitivity to disturbances and performance is a common tradeoff in control design.

Figures 6 and 7 illustrate the behavior of this algorithm for under the exact same conditions of figures 4 and 5 with $\kappa=0.675$. If one assumes a $\delta=1.175$ then the algorithm allows all of the links to maintain their minimum SIR's with the addition of the fifth user.

In both the basic Foschini-Miljanic algorithm and the DPC/ALP of Bambos et al there is a single gain parameter 

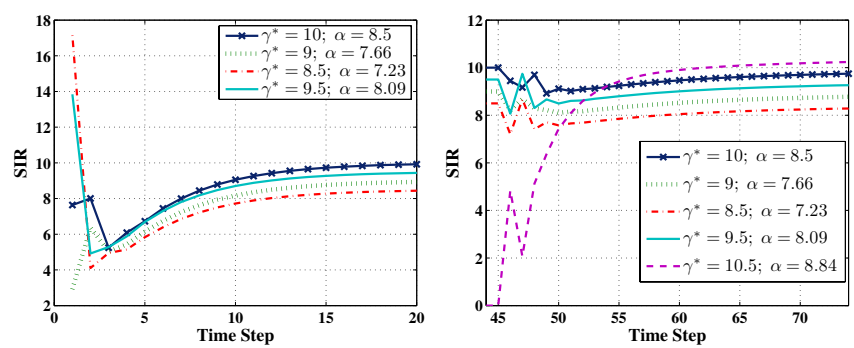

Fig. 6. Startup using the $\gamma$ algorithm from equation (15).

Fig. 7. Adding a new user with the $\gamma$ algorithm from equation (15).

$(\gamma)$ associated both the transient properties and the equilibrium properties (target SIR). A more general approach is to introduce some means to 'decouple' the transients from the steady state control mechanisms, in order to affect the behavior during the transient and equilibrium phases separately. For example, one could consider a new time varying gain parameter for the noise term (as a gain for transient behavior) as well as the time varying $\gamma(k)$ introduced above. The power update scheme would then be

$$
\mathbf{p}(k+1)=D_{\gamma(k)} F \mathbf{p}(k)+D_{\beta(k)} \mathbf{u}
$$

where $D_{\gamma(k)}$ and $D_{\beta(k)}$ are both time dependent gain matrices with each link's $\gamma_{i}(k)$ and $\beta_{i}(k)$ on their diagonal. Overall, numerical experience indicates that we can significantly improve the transient behavior by the appropriate choice of update algorithm while maintaining the autonomous nature of updates and ease of operation.

\section{CONCLUSIONS AND EXTENSIONS}

While equilibrium properties of iterative power control algorithms are well studied, understanding transients and designing for transients are as important yet much less explored. In this paper, we began to address this issue by bounding SIR transients using invariant sets and their properties for the basic DPC algorithm by Foschini and Miljanic. We provided a sufficient condition to guarantee that the SIR for all links will not dip below a minimum threshold. Further, we showed that verifying the invariance of certain SIR regions for this algorithm can be done via LP, through a systematic and efficient computational approach. We also presented preliminary design results based on introducing a time varying gain parameter. This control design can be analyzed with the tools introduced herein.

Our results can readily be generalized to applications where the SIR levels can be allowed to drop below their minimum temporarily. In this case one can guarantee that after $T$ time steps SIR's can be restored to the desired level using similar analysis.

Transients of iterative network resource allocation are generally under-explored so there are many directions for future work in the areas of analysis, computational approach and control design. On the analysis side, an important direction for future work is to obtain necessary conditions for SIR guarantees discussed in section (III-A). The computational method for invariance analysis can also be developed further by noting that LP is a special case of a far more general computational approach for verifying the invariance of wide range of SIR regions for a variety of nonlinear power updates. In control design the next step is to tune the update algorithm for the $\gamma_{i}(k)$ 's and study the effect of different update algorithms for the both the $\gamma_{i}(k)$ 's and the $\beta_{i}(k)$ 's described in section VI.

\section{ACKNOWLEDGMENT}

This work has been supported in part by NSF Grants CCF0448012, CNS-0417607, and AFOSR FA9550-06-1-0297. The authors would like to thank Prashanth Hande at Qualcomm and Princeton University for his helpful comments.

\section{REFERENCES}

[1] M. Andersin. Power Control and Admission Control in Cellular Radio Systems. PhD thesis, Royal Institute of Technology, Sweden, 1996.

[2] M. Andersin, Z. Rosberg, and J. Zander. Soft and safe admission control in cellular networks. IEEE/ACM Transactions on Networking, 5(2), Apr. 1997.

[3] N. Bambos, S. C. Chen, and G. J. Pottie. Channel access algorithms with active link protection for wireless communication networks with power control. IEEE/ACM Tran. Networking, 8(5):583-597, Oct. 2000.

[4] S. Boyd and L. Vandenberghe. Convex Optimization. Cambridge University Press, 2004.

[5] G. J. Foschini and Z. Miljanic. A simple distributed autonomous power control algorithm and its convergence. IEEE Tran. on Vehicular Technology, 42(3):641-646, Nov. 1993.

[6] P. Hande, S. Rangan, and M. Chiang. Distributed algorithms for optimal QoS assignment in wireless cellular data networks. In Proc. IEEE Infocom, Barcelona, Spain, April 2006.

[7] D. Mitra. An asynchronous distributed algorithm for power control in cellular radio systems. In Proc. 4th WINLAB Workshop on 3rd Generation Wireless Information Networks, 1994.

[8] D. Mitra and J. A. Morrison. A Distributed Power Control Algorithm for Bursty Transmission on Cellular, Spread Spectrum Wireless, pages 201-212. Kluwer, 1996. editor: J.M. Holtzman.

[9] D. Mitra and J. A. Morrison. A Novel Distributed Power Control Algorithm for Classes of Service in Cellular CDMA Networks, pages 187-202. Kluwer, 1998. editors: J.M.Holtzman and M.Zorzi.

[10] P. A. Parrilo. Sum of squares programs and polynomial inequalities. SIAG/OPT Views-and-News: A Forum for the SIAM Activity Group on Optimization, 15(2):7-15, October 2004.

[11] J. F. Strum. Using sedumi 1.02, a matlab toolbox for optimization over symmetric cones (updated for version 1.05). Optimization Methods and Software, 11-12:625-653, 1999 (for verions 1.02/1.03). Updated in 2001 and available from http://sedumi.mcmaster.ca.

[12] S. Wiggins. Introduction to Applied Nonlinear Dynamical Systems and Chaos. Texts in Applied Mathematics. Springer-Verlag, 2nd edition, 2003

[13] R. Yates. A framework for uplink power control in cellular radio systems. IEEE J. Sel. Areas Commun, 13(7):1341-1348, Sept. 1995.

[14] R. Yates and C. Y. Huang. Integrated power control and base station assignment. IEEE Trans. on Vehicular Technology, 44(3):638-644, Aug 1995. 